\title{
Electron ptychography using an ultrafast direct electron detector
}

Biying Song ${ }^{1}$, Zhiyuan Ding ${ }^{1}$, Christopher Allen ${ }^{2,3}$, Hidetaka Sawada ${ }^{4}$, Xiaoqing Pan ${ }^{5}$, Angus. I. Kirkland $^{2,3}$, and Peng Wang ${ }^{1 *}$

${ }^{1}$ National Laboratory of Solid State Microstructures, College of Engineering and Applied Sciences and Collaborative Innovation Center of Advanced Microstructures, Nanjing University, Nanjing 210093, People's Republic of China.

${ }^{2}$ Department of Materials, University of Oxford, Parks Road, Oxford OX1 3PH, UK.

${ }^{3}$ Electron Physical Sciences Imaging Centre, Diamond Lightsource Ltd., Diamond House, OX11 0DE, U.K.

${ }^{4}$ JEOL Ltd, 1-2 Mushashino, 3-Chome, Akishima, Tokyo 196, Japan.

${ }^{5}$ Department of Chemical Engineering and Materials Science and Department of Physics and Astronomy, University of California, Irvine, CA 92697, USA.

* Corresponding author: wangpeng@nju.edu.cn

In recent years, scanning transmission electron microscopy (STEM) has become an important research tool for materials characterization. Using an annular detector geometry, electron energy loss spectroscopy (EELS) can be combined with high-angle annular dark field (HAADF) imaging to provide simultaneous structural and compositional information [1]. However, due to the dependence on atomic number of $\mathrm{Z}$ contrast, HAADF imaging is relatively insensitive to light elements [2]. By comparison, ptychography, as a phase sensitive imaging technique, has attracted great interest due to its high sensitivity to light elements at atomic resolution, especially given recent developments in fast direct-electron detectors and data-processing algorithms [3-6]. The phase recovered from the exit wave can simultaneously image light and heavy atoms [4] and further be extended to provide information along the Z-axis [3,5]. Furthermore, as the theoretical resolution is determined by the maximum spatial frequency recorded in diffraction patterns, electron ptychography has the potential to recover super-resolution phase data [6]. However, as entire forward-scattered electrons have to be detected for phase reconstruction, no electrons are allowed to enter an EELS spectrometer. Therefore, a method compatible with quantitative phase recovery and simultaneous EELS analysis has not been demonstrated.

In this work, we will briefly demonstrate the capabilities of defocused ptychography to achieve a 2D phase reconstruction of nanocrystals at sub-ångström resolution [4] and 3D reconstruction of nanostructured materials [5]. Subsequently, we propose a new geometry for iterative ptychographic reconstruction from synthesized hollow diffraction patterns using a focused scanned probe [7]. This 5D-STEM imaging method with a hollow direct-electron detector not only fully integrates various traditional STEM imaging modes, including HAADF, annular bright field and differential phase contrast, as well as X-ray energy dispersive spectrometry and EELS mapping, but also provides ptychographic reconstructed phases with higher resolution and contrast for light elements.

Fig. 1(a) shows a schematic illustration of the 5D-STEM experimental setup. JEM-ARM300F electron microscope was operated at $80 \mathrm{kV}$ with a convergence semi-angle of $24 \mathrm{mrad}$. The focused probe was raster-scanned across monolayer $\mathrm{MoS}_{2}$ as shown in Fig. 1(b) with $256 \times 256$ probe positions and with a step size of $0.204 \AA$. The images shown in Fig. 2, demonstrate that the phase of monolayer $\mathrm{MoS}_{2}$ reconstructed from hollow diffraction patterns can achieve a resolution of $0.91 \AA$ and that the $2 \mathrm{~S}$ sites 
are clearly resolved. In order to match the experimental requirements for atomic-resolution EELS mapping, we have explored the effects of scan step sizes and various hollow inner angles on the reconstruction using hollow ptychography. As the dark field disk signals in diffraction patterns were used to reconstruct the phase, the missing information in the center of each diffraction pattern can be retrieved. Therefore, if fast hollow detectors are developed that are compatible with an EELS spectrometer, we anticipate that this geometry will enable simultaneous, correlative analysis using both highly sensitive phase information and chemical mapping.

\section{References:}

[1] K. Kimoto, et al., Nature 450 (2007), 702.

[2] P. D. Nellist, et al., Science 305 (2004), 1741.

[3] H. Yang, et al., Nature Communications 7 (2016), 12532.

[4] P. Wang, et al., Scientific Reports 7 (2017), 2857.

[5] S. Gao, et al., Nature Communications 8 (2017), 163.

[6] Y. Jiang, et al., Nature 559 (2018), 343.

[7] B. Song, et al., Physical Review Letters, 121 (2018), 146101.

[8] The authors acknowledge funding from the National Natural Science Foundation of China (11874199), and the National Basic Research Program of China, (Grant No. 2015CB654901).

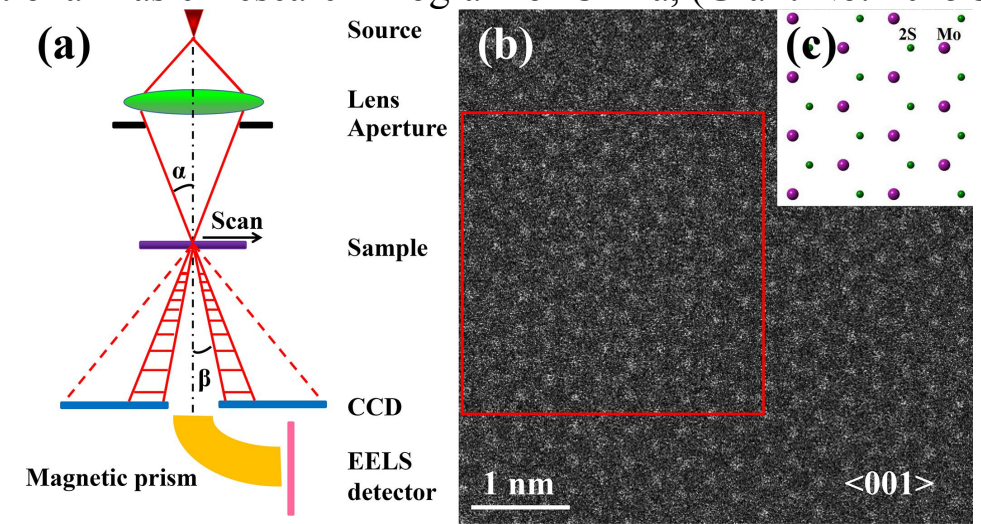

Figure 1. (a) Schematic of the experimental configuration used for ptychographic reconstruction. (b) HAADF image of monolayer $\mathrm{MoS}_{2}$ oriented along a $<001>$ direction. (c) Projected atomic model.
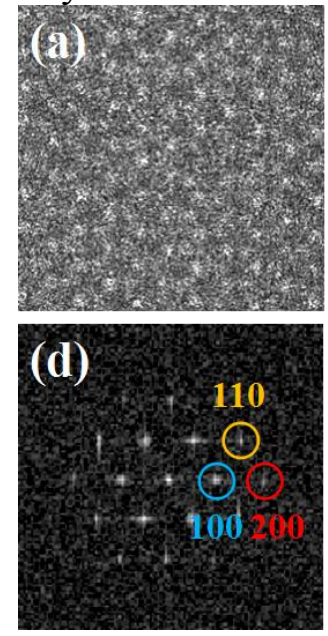
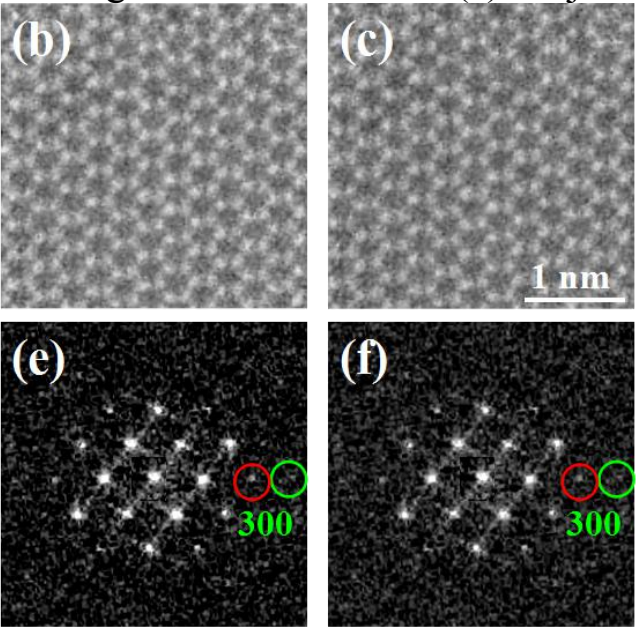

Figure 2. (a) ADF image of monolayer $\mathrm{MoS}_{2}$. (b) Conventional and (c) Hollow ptychographic ( $\beta=5$ $\mathrm{mrad})$ reconstructed phase. $(\mathrm{d}-\mathrm{f})$ Power spectra of $(\mathrm{a}-\mathrm{c})$ respectively. 